\title{
Magnetic resonance imaging as an adjunct to computed tomography in the diagnosis of pulmonary Hydatid cysts
}

\author{
Roopa Tandur, Aparna Irodi, Binita Riya Chacko, Leena Robinson Vimala, \\ Devasahayam Jesudas Christopher', Birla Roy Gnanamuthu² \\ Departments of Radiodiagnosis, ${ }^{1}$ Pulmonary Medicine and ${ }^{2}$ Cardiothoracic Surgery, Christian Medical College, Vellore, Tamil Nadu, India \\ Correspondence: Dr Aparna Irodi, Department of Radiodiagnosis, Christian Medical College, Vellore, Tamil Nadu, India. \\ E-mail: aparnashyam@gmail.com
}

\begin{abstract}
Introduction: Although pulmonary hydatid cysts can be diagnosed on computed tomography (CT), sometimes findings can be atypical. Other hypodense infective or neoplastic lesions may mimic hydatid cysts. We proposed that magnetic resonance imaging (MRI) may act as a problem-solving tool, aiding the definite diagnosis of hydatid cysts and differentiating it from its mimics. The aim of this study is to assess the findings of pulmonary hydatid cysts on CT and MRI and the additional contribution of MRI in doubtful cases. Materials and Methods: This is a retrospective study of 90 patients with suspected hydatid cysts. CT and MRI findings were noted and role of MRI in diagnosing hydatid cysts and its mimics was studied. Descriptive statistics for CT findings and sensitivity and specificity of MRI were calculated using surgery or histopathology as gold standard. Results: Of the 90 patients with suspected pulmonary hydatid cysts, there were 52 true-positive and 7 false-positive cases on CT. Commonest CT finding was unilocular thick-walled cyst. In the 26 patients who had additional MRI, based on T2-weighted hypointense rim or folded membranes, accurate preoperative differentiation of 14 patients with hydatid cysts from 10 patients with alternate diagnosis was possible. There was one false-positive and one false-negative case on MRI. Conclusion: Although hydatid cyst can be diagnosed on CT on most occasions, sometimes there are challenges with certain mimics and atypical appearances. T2-weighted MRI can act as a problem solving tool to conclusively diagnose hydatid cyst or suggest an alternate diagnosis.
\end{abstract}

Key words: Computed tomography; hydatid; magnetic resonance imaging; pulmonary

\section{Introduction}

Cysts and cyst-like hypodense lung lesions sometimes pose diagnostic challenge on chest computed tomography (CT). In endemic areas, hydatid cyst is one of the top differential diagnoses. Tumors like schwannoma with cystic degeneration, ${ }^{[1]}$ sinister lesions like necrotic lung cancer, ${ }^{[2]}$ metastases, ${ }^{[3]}$ and infections like tuberculosis may mimic hydatid cyst.

\begin{tabular}{|l|l|}
\hline \multicolumn{2}{|c|}{ Access this article online } \\
\hline Quick Response Code: & \\
\cline { 1 - 2 } & Website: \\
& www.ijri.org \\
\cline { 2 - 3 } & DOI: \\
& \\
\hline
\end{tabular}

When hydatid cysts show typical appearances like crescent sign, onion peel, or cumbo sign or folded membranes, the diagnosis is straightforward. However, atypical appearances with ruptured, collapsed, or infected cysts showing more hyperdense or solid appearance can pose problems, mimicking infections like tuberculosis or neoplastic lesions. We propose that in such situations, magnetic resonance

This is an open access journal, and articles are distributed under the terms of the Creative Commons Attribution-NonCommercial-ShareAlike 4.0 License, which allows others to remix, tweak, and build upon the work non-commercially, as long as appropriate credit is given and the new creations are licensed under the identical terms.

For reprints contact: reprints@medknow.com

Cite this article as: Tandur R, Irodi A, Chacko BR, Vimala LR, Christopher DJ, Gnanamuthu BR. Magnetic resonance imaging as an adjunct to computed tomography in the diagnosis of pulmonary Hydatid cysts. Indian J Radiol Imaging 2018;28:342-9. 
imaging (MRI) may play a problem-solving role. The aim of the study is to describe the findings of pulmonary hydatid cysts on chest radiograph, CT, and MRI and study the role of MRI in patients with uncertain findings on CT.

\section{Materials and Methods}

We retrospectively analyzed CT thorax of 90 patients, performed between February 2006 and January 2018, with suspected or confirmed pulmonary hydatid cysts. All the patients had undergone chest radiograph, contrast-enhanced CT thorax with $2.5-5 \mathrm{~mm}$ axial sections, and coronal reconstructions. Patients with uncertain findings on CT had undergone additional axial and coronal T2-weighted fast/turbo spin-echo nonrespiratory-gated MRI. MRI was performed within a week of the CT. Time taken for the sequences was $5 \mathrm{~min}$.

The CT and MRI findings were studied and compared with the final diagnosis based on histopathology. Descriptive statistics were used to list the imaging findings. Sensitivity and specificity of MRI were calculated. We also assessed the chest radiographic findings of confirmed pulmonary hydatid cysts.

\section{Results}

Of the total 90 patients suspected on the basis of CT to have pulmonary hydatid cyst, 67 patients were finally diagnosed to have hydatid cysts, 18 patients had alternate diagnoses, and 5 patients were lost to follow-up. Figure 1 shows details of the study cohort and mode of diagnosis.

Findings of hydatid cysts diagnosed on CT

A total of 60 cysts were seen in 52 true-positive cases diagnosed on CT alone (excluding 3 cases with multiple cysts). Details of the CT findings are given in Table 1. Most of the cysts $(n=28)$ were seen as mildly thick-walled homogeneous unilocular cysts [Figure 2]. Thin imperceptible wall was seen in only a few cysts $(n=5)$. Some of the cysts showed crescent sign $(n=5)$ [Figure 3], air bubble sign $(n=9)$ [Figure 4 ], and folded membrane appearance $(n=13)$ [Figure 5]. Daughter cyst was seen in one patient. False-positive diagnosis of hydatid cyst was made on CT in seven patients based on the cystic appearance, of whom three had abscesses and the other four had endometriotic cyst, congenital cystic adenomatoid malformation, epithelial cyst, and gastroenteric cyst.

\section{MRI findings}

Twenty-six patients had additional T2-weighted MRI in an attempt to further characterize the lesions with uncertain findings on CT. High attenuating or enhancing areas, calcification, thick irregular septae, abscess like appearance, mediastinal adenopathy, hydropneumothorax, and filling defects in pulmonary arteries in a patient with past history of hydatid cyst were some of the findings which prompted performing the additional MRI. In a few instances, additional MRI was done in purely cystic lesions to look for the hypointense rim.

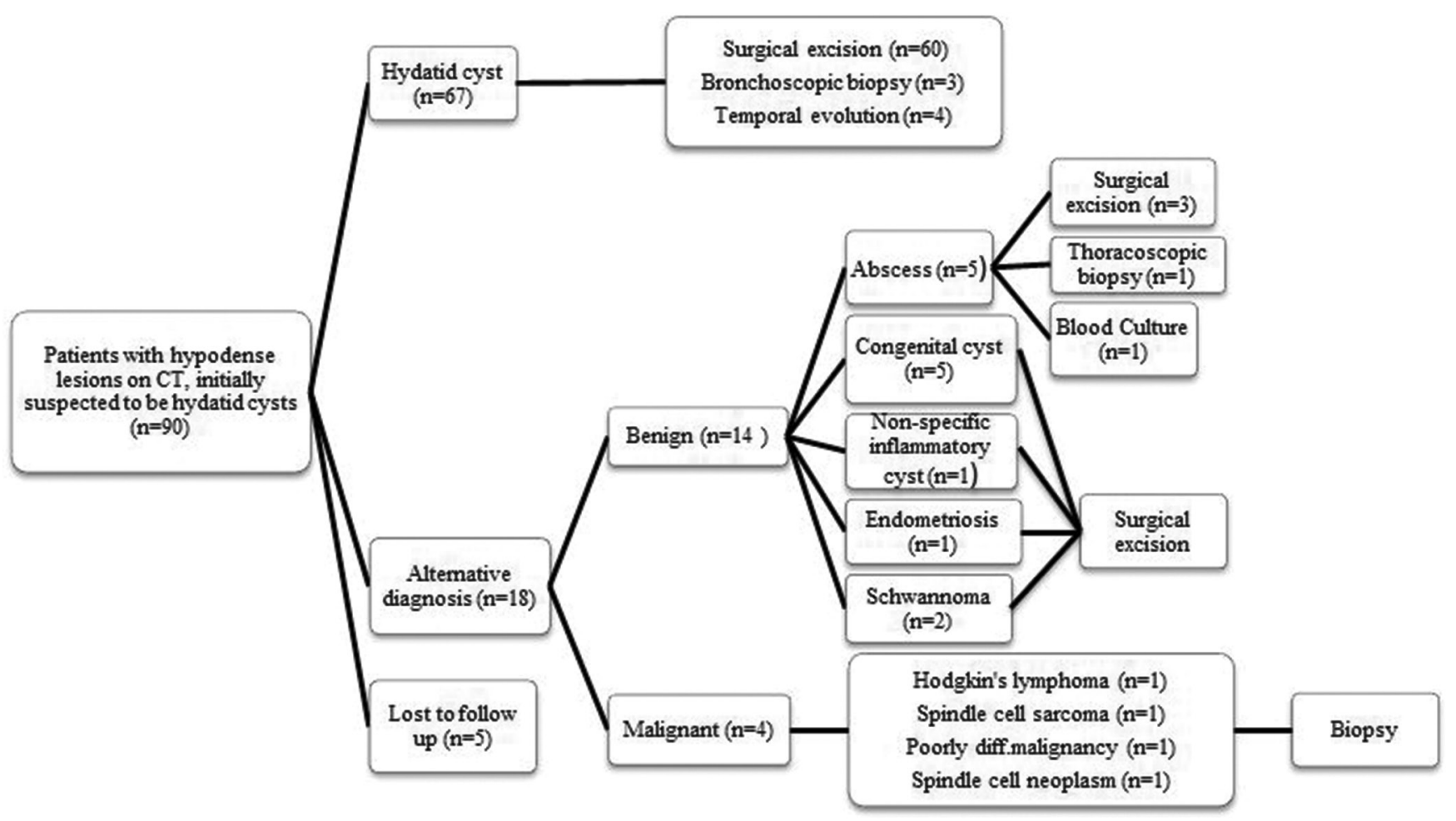

Figure 1: Final diagnosis in the study cohort of patients with hydatid cyst suspected on computed tomography with the mode of establishing diagnosis 


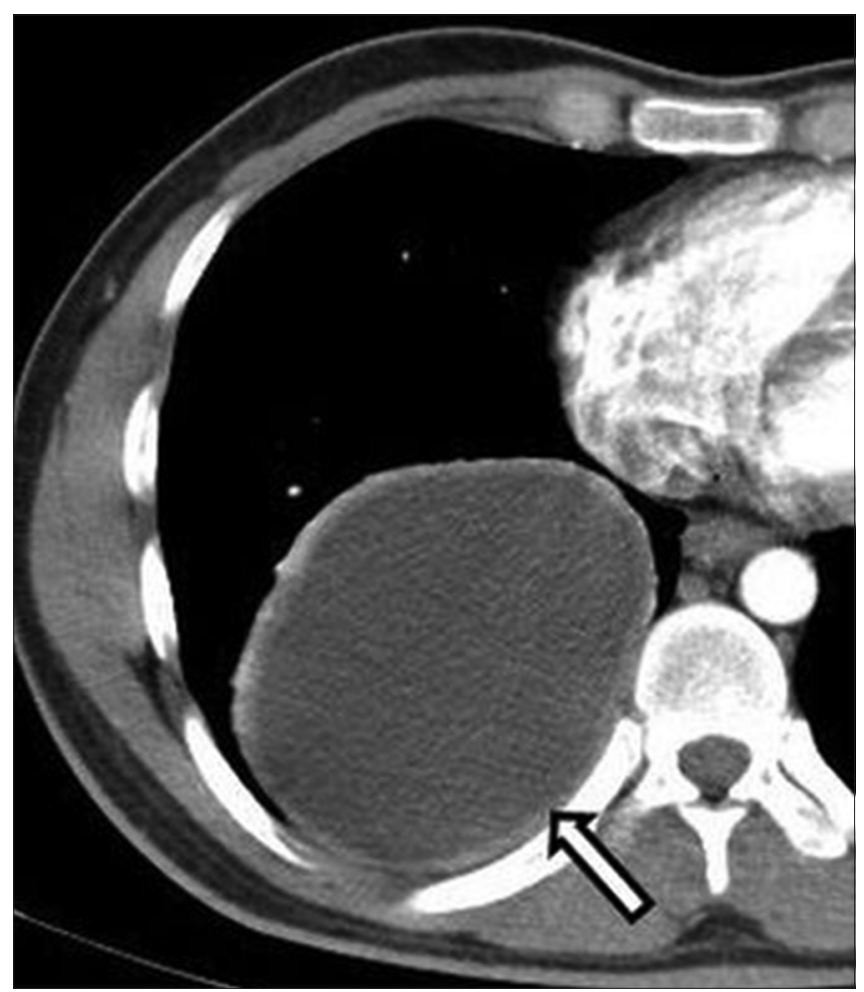

Figure 2: Contrast-enhanced axial computed tomography of the chest in mediastinal window showing well-defined unilocular thick-walled (arrow) cystic lesion in the right lower lobe

Table 1: Imaging features of proven hydatid cysts diagnosed on computed tomography

\begin{tabular}{lc}
\hline $\begin{array}{l}\text { Appearance of proven hydatid } \\
\text { cysts on CT }\end{array}$ & $\begin{array}{c}\text { Number of cysts (80 cysts in } \\
\text { patients excluding } 4 \text { cases with } \\
\text { innumerable cysts) }\end{array}$ \\
\hline Thick wall unilocular cyst & 28 \\
Thin wall unilocular cyst & 7 \\
Air crescent sign & 5 \\
Air bubble sign & 9 \\
Folded membrane & 13 \\
High attenuation areas & 5 \\
Calcification & 1 \\
Mimicking infection/abscess & 5 \\
(thick wall with surrounding & \\
consolidation) & 3 \\
Mimicking mass (solid appearing) & 1 \\
Presence of satellite cysts & 1 \\
Cavity with thick irregular septae & \\
and associated mediastinal lymph & \\
nodes & 1 \\
Hydropneumothorax & \\
Filling defects in pulmonary artery & \\
Total number of cysts & \\
\hline
\end{tabular}

CT: Computed tomography

On T2-weighted MRI, there were 14 true-positive cases with 18 hydatid cysts (excluding 1 case showing numerous cysts). Figure 6 shows the details of patients in whom

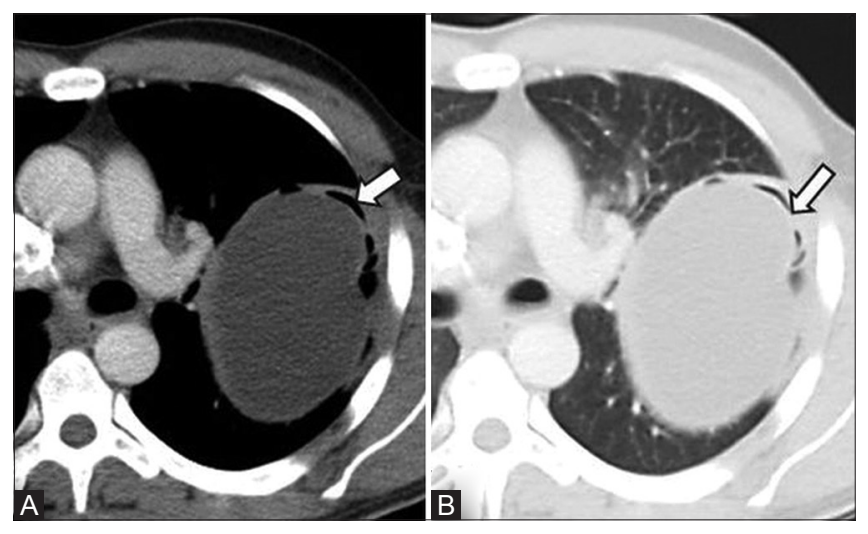

Figure 3 ( $\mathrm{A}$ and $\mathrm{B}$ ): Contrast-enhanced axial computed tomography of the chest in mediastinal $(A)$ and lung window $(B)$ showing hydatid cyst in the left upper lobe with air crescent (white arrow)

additional MRI was performed. Hypointense folded membranes [Figures 7 and 8 ] aided the diagnosis of hydatid cyst in 16 cysts (89\%) and hypointense wall (hypointense rim sign) [Figure 9] helped in diagnosis of 2 unruptured cysts $(11 \%)$. In one patient with past history of surgery for pericardial hydatid cyst, CT showed a lung cyst and also hypodense filling defects with focal dilatation of a few peripheral pulmonary artery branches [Figure 10]. Hydatid embolism into the pulmonary arteries was considered which was confirmed on T2-weighted MRI, which showed tiny cystic lesions with hypointense membranes within the pulmonary artery branches [Figure 10].

Of the 10 patients who were true negative on MRI, 7 patients showed solid and cystic areas without folded membranes or hypointense rim [Figure 11] suggesting an alternate diagnosis and prompting image guided biopsy. Four of these were malignant lesions and three were benign [Figure 6]. In the remaining three patients, well-defined cysts with thin wall [Figure 12] and the lack of folded membranes suggested alternate cystic lesions. These patients after surgical resection proved to be having pleuropericardial cyst, enteric cyst, and a nonspecific benign cyst.

In one patient with a fungal infection, the debris on MRI resembled folded membranes, resulting in a false-positive diagnosis of hydatid cyst [Figure 13]. In another patient with surgically proven hydatid cyst, hypodense lesion with faint peripheral nodular calcification was seen on CT and the MRI did not show any folded membranes leading to a false-negative diagnosis of neoplastic lesion preoperatively [Figure 14]. The sensitivity, specificity, positive predictive value, and negative predictive value of MRI in diagnosis of hydatid cyst were $93 \%$, $91 \%, 93 \%$, and $91 \%$, respectively.

Number, size, and distribution of hydatid cysts

Single cyst $(80 \%)$ was more common than multiple cysts. Size of the cysts varied from 2 to $18 \mathrm{~cm}$. Hydatid cysts were more common in the middle and lower lobes $(75 \%)$, right lung $(60 \%)$ being slightly more affected than the left. Pleural 


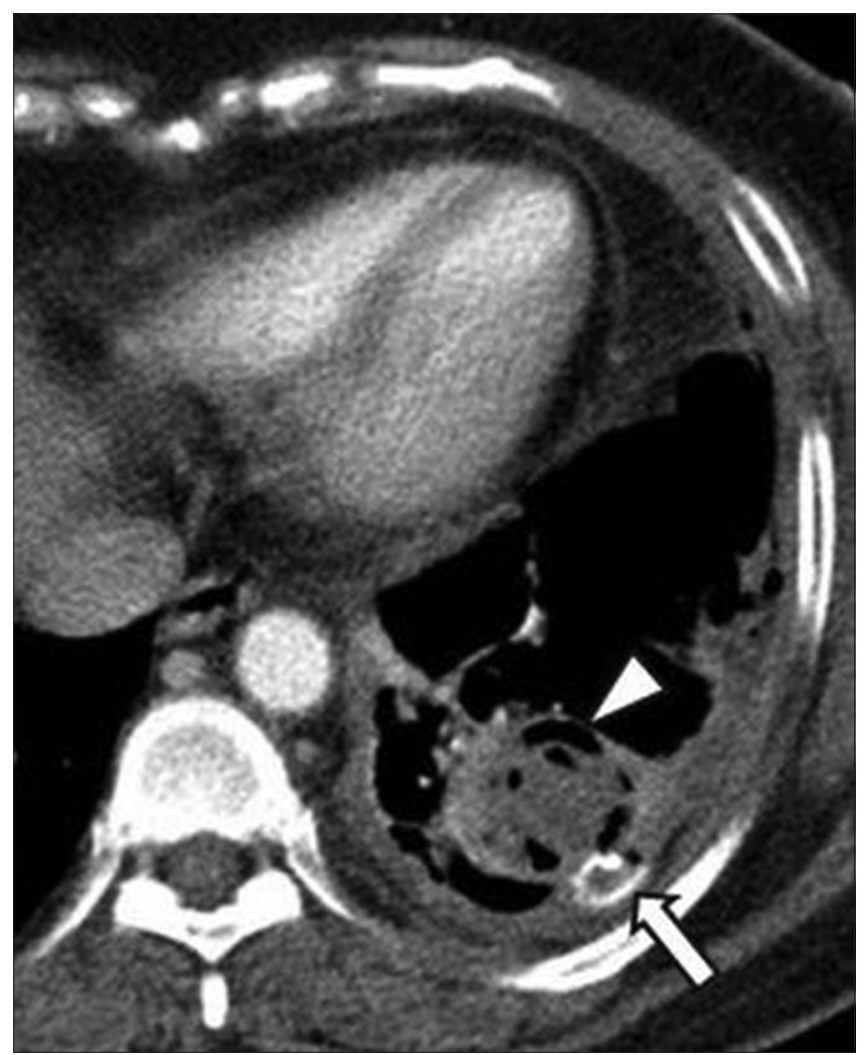

Figure 4: Contrast-enhanced axial computed tomography of the chest in mediastinal window showing air bubble sign (arrowhead) in a left lower lobe hydatid cyst. This patient also had an associated pleural effusion with intercostal drain (white arrow)

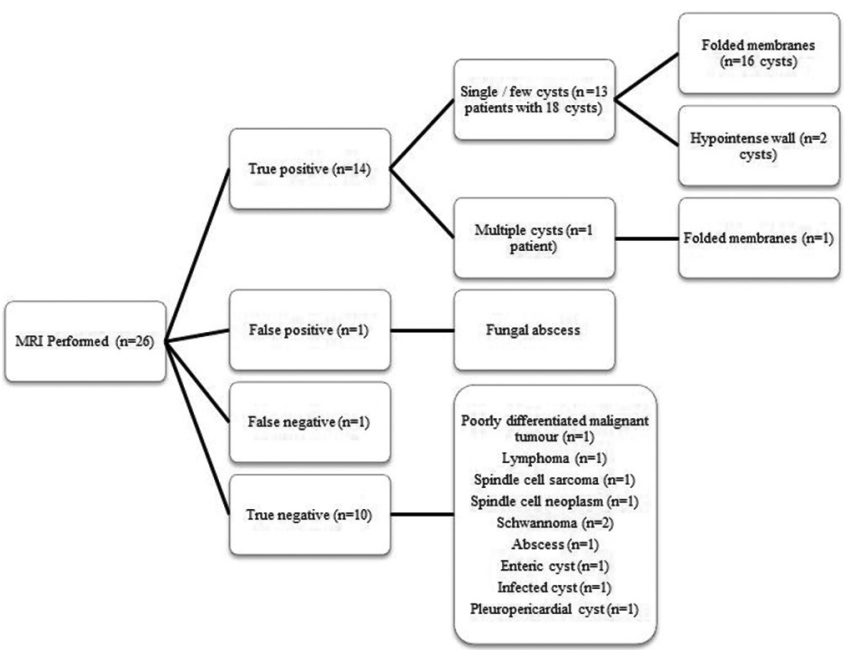

Figure 6: Flowchart showing the details of patients in whom additional magnetic resonance imaging was performed

cysts were seen in six patients. Concurrent liver cysts were seen in 12 patients. One patient showed additional hydatid cyst in the adrenal gland and another patient showed additional hydatid cyst in the neck.

Chest radiographic findings of hydatid cysts

In 67 patients with 77 hydatid cysts (excluding the numerous cysts which were practically not countable), 76 cysts could

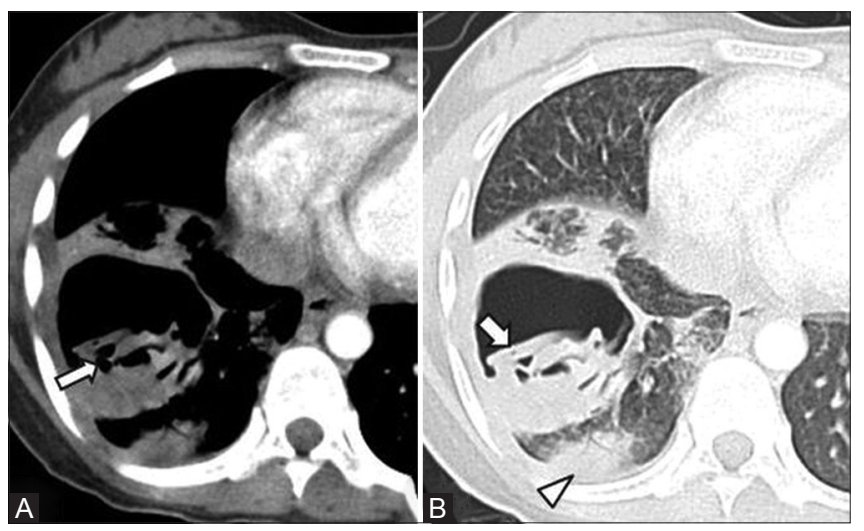

Figure 5 (A and B): Axial contrast-enhanced computed tomography of the chest in mediastinal $(A)$ and lung window (B) show a ruptured hydatid cyst in the right lower lobe with folded membranes (white arrow). Note consolidation in the adjoining lung (arrowhead)
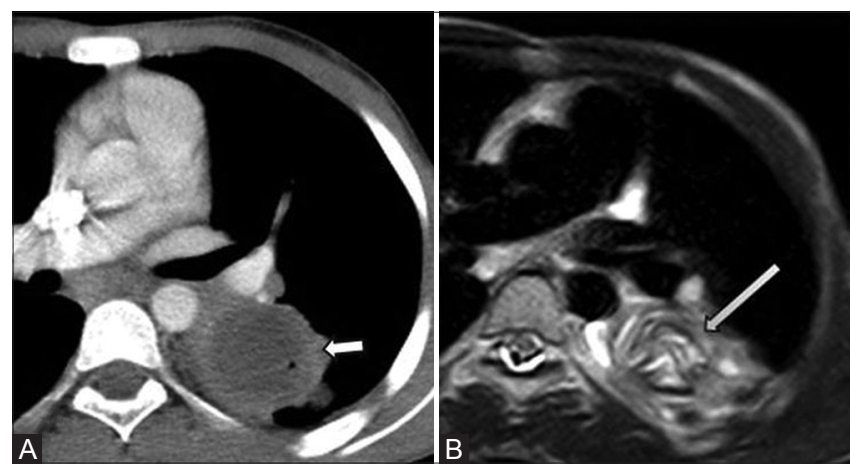

Figure 7 (A and B): Contrast-enhanced axial computed tomography of the chest $(A)$ showing well-defined hypodense lesion in the left lower lobe with thick wall (white arrow) and surrounding consolidation, where hydatid cyst and abscess were considered. Axial T2-weighted image (B) demonstrates hypointense folded membranes (long white arrow) helping to make the diagnosis of hydatid cyst

be seen on chest radiograph. Of these, 39 cysts were well defined with round [Figure 15A] or oval shape [Figure 15B]. Five cysts were lobulated [Figure 15C]. Sixteen cysts were ill defined with nonspecific appearance. Two cysts presented as consolidation. Folded membranes [Figure 15D] were seen in seven cysts and crescent sign [Figure 15E] was seen in five cysts. Pleural effusion and hydropneumothorax were seen in chest radiograph in one patient each [Figure 15F].

\section{Ultrasound findings}

Ultrasound played a complementary role in the evaluation of five true-positive cases of hydatid cysts on CT which were located peripherally. Collapsed membranes [Figure 16] were identified in three of these patients and trilaminar wall in two patients.

\section{Discussion}

Hydatid cyst is a parasitic infection caused by Echinococcus granulosus. Humans are the accidental hosts. ${ }^{[4]}$ Hydatid cyst consists of three layers with fluid within. The outermost layer called the pericyst or ectocyst is formed by the host 


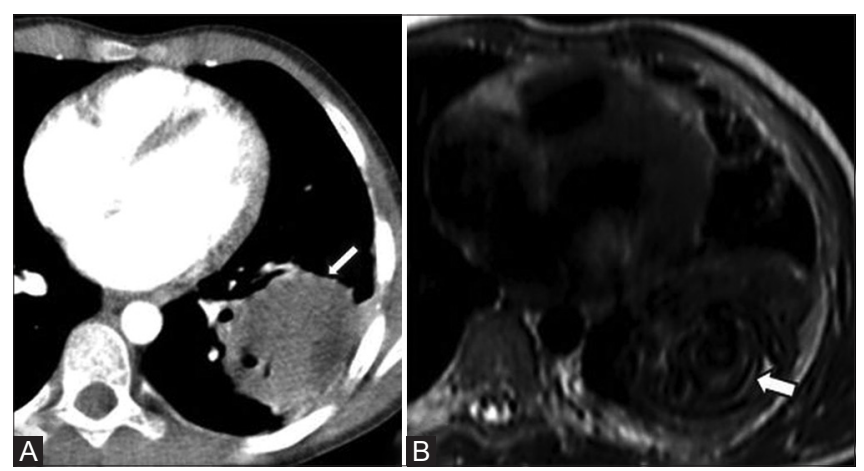

Figure $\mathbf{8}$ (A and B): Contrast-enhanced axial computed tomography scan of chest $(A)$ showing an irregular hypodense lesion in the left lower lobe (arrow). Neoplastic lesion was initially considered on computed tomography. Axial T2-weighted magnetic resonance imaging (B) showing hypointense folded membranes (white arrow) aiding accurate preoperative diagnosis of hydatid cyst

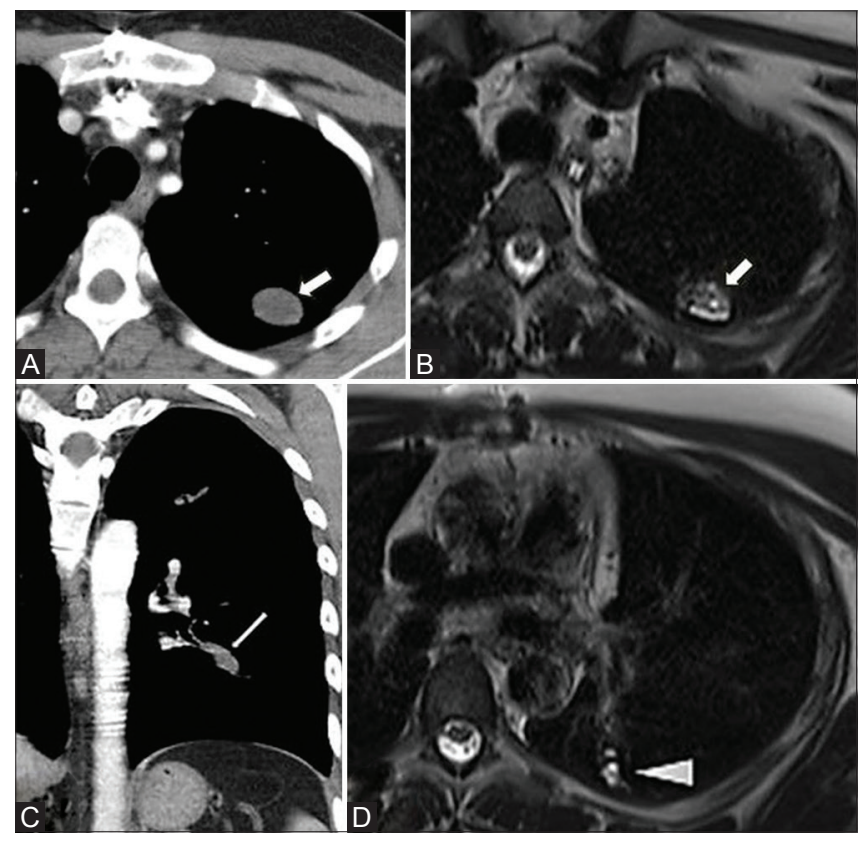

Figure 10 (A-D): Axial contrast-enhanced computed tomography of thorax (A) showing a small ovoid hypodense lesion in the left upper lobe. Axial T2-weighted magnetic resonance imaging images (B) showing hypointense folded membranes confirming the diagnosis of hydatid cyst. This patient also had filling defects in the pulmonary arteries (C) which were hyperintense on axial T2-weighted magnetic resonance images with small membranes (D), confirming hydatid embolization

tissue's reaction to parasites. The middle layer is the laminated membrane or exocyst and the innermost layer is known as the germinative layer or endocyst. ${ }^{[5]}$ Liver is the most common organ $(60 \%)$ to be affected in adults, followed by lung (20-30\%). ${ }^{[6]}$ In children, lungs are more commonly involved. . $^{[7-9]}$

Patients with pulmonary hydatid cysts may be asymptomatic or present with symptoms like cough, hemoptysis, and chest pain. ${ }^{[10,11]}$ Patients may even present with acute onset cough, hemoptysis, and anaphylactic reaction during rupture of cyst. ${ }^{[11]}$

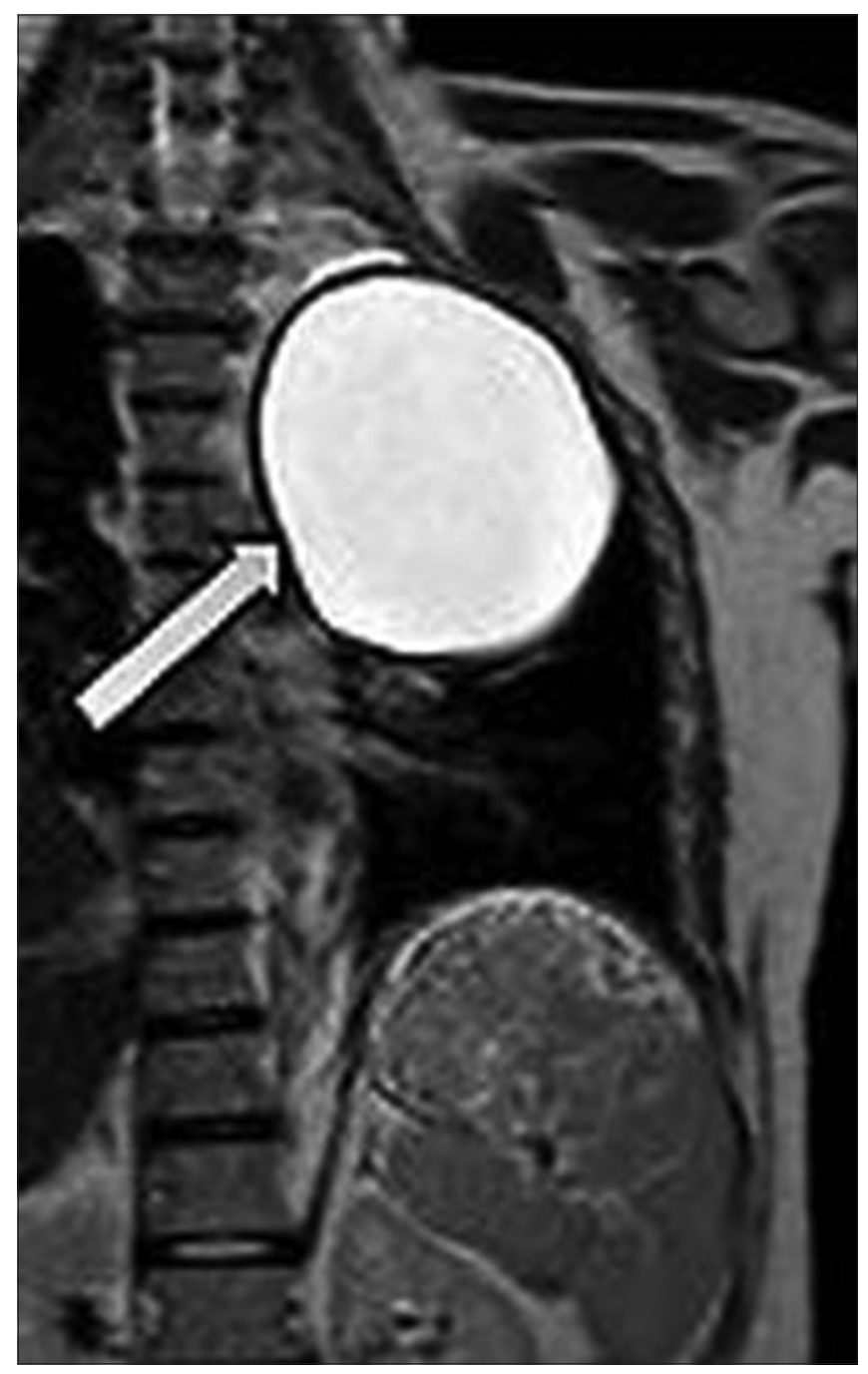

Figure 9: Coronal T2-weighted magnetic resonance imaging image showing hyperintense cystic lesion in the left upper lobe with hypointense rim sign

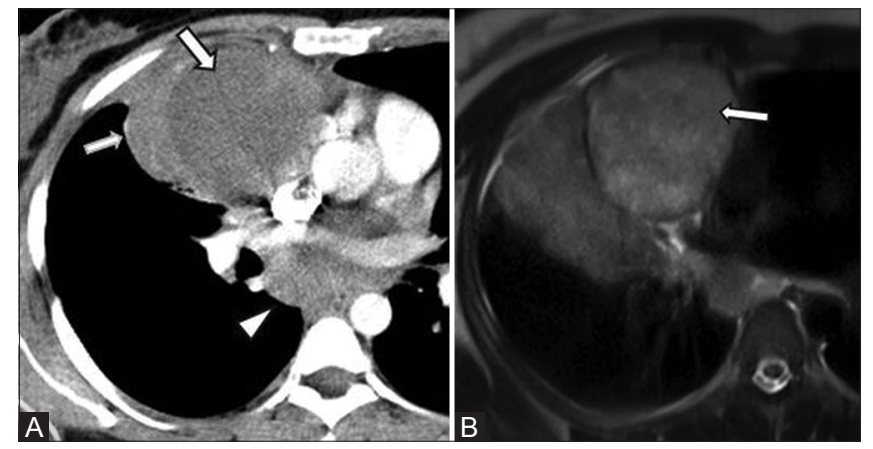

Figure 11 (A and B): Contrast-enhanced axial computed tomography of the chest $(A)$ showing a hypodense lesion in the right middle lobe (white arrow) with surrounding consolidation (small arrow) and mediastinal adenopathy (arrowhead). Abscess and hydatid cyst were considered. T2-weighted axial image (B) showed heterogeneous solid areas in the lesion (white arrow) suggesting a neoplastic lesion. Computed tomography-guided biopsy showed lymphoma

On CT, unruptured hydatid cyst appears as well-defined homogeneous cystic lesions which are difficult to 


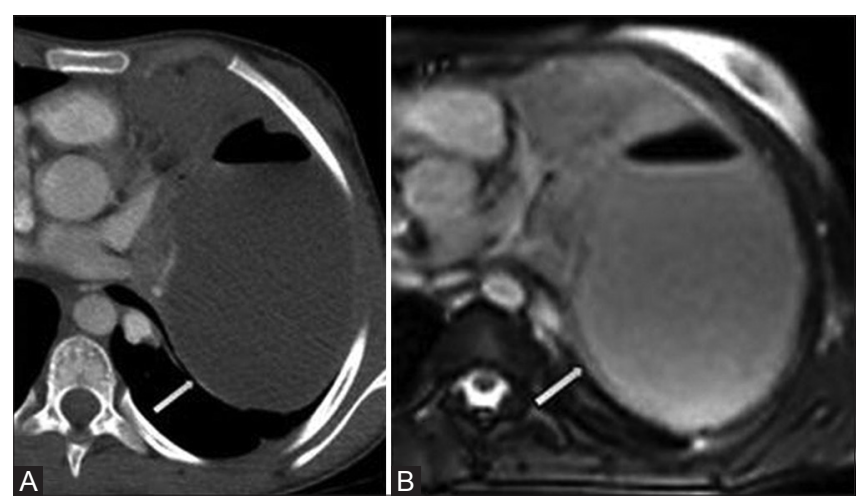

Figure 12 (A and B): Contrast-enhanced axial computed tomography of chest $(A)$ showing well-defined thin-walled cystic lesion (white arrow) with air-fluid level in the left lung. Hydatid cyst, infected intrapulmonary bronchogenic cyst, and congenital cystic adenomatoid malformation (CCAM) were considered on computed tomography. T2-weighted axial magnetic resonance image (B) showing well-defined thin-walled hyperintense cystic lesion (arrow) with air-fluid level. Note the absence of hypointense rim or folded membranes. Patient underwent surgical excision and the lesion turned out to be a nonspecific benign cyst
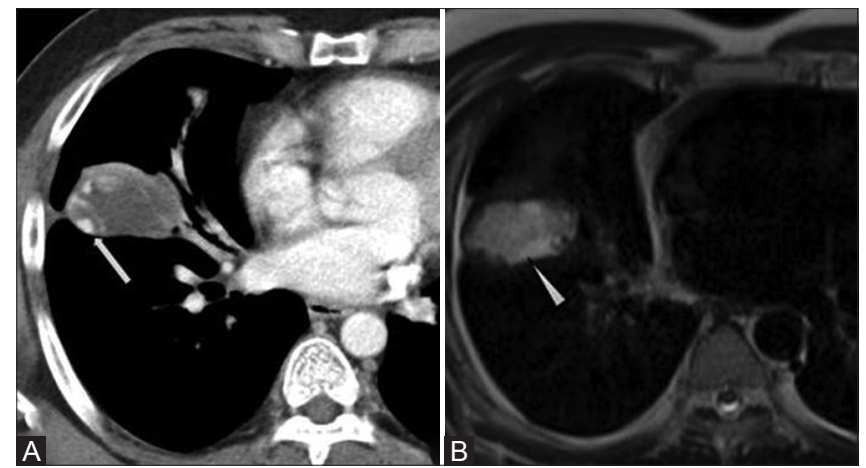

Figure 14 (A and B): Axial contrast-enhanced computed tomography of thorax (A) showing a hypodense lesion with few peripheral calcifications (long white arrow). Due to the presence of calcifications, hydatid cyst was thought less likely. However because of the hypodense appearance, T2-weighted axial magnetic resonance imaging (B) was done which also did not show hypointense wall or folded membranes, precluding an imaging diagnosis of hydatid cyst. This lesion was finally diagnosed to be a hydatid cyst after surgery

differentiate from other cysts, apart from mildly thicker hyperdense walls. ${ }^{[12]}$ Different types of CT appearances have been described in ruptured hydatid cysts. Cyst growth can erode the adjacent bronchioles and air can track between the pericyst and exocyst giving rise to the meniscus or crescent sign, which some consider as a sign of impending rupture. When air continues to enter the cyst, exocyst ruptures and parallel arches of air can be seen between the pericyst and exocyst named as onion peel or cumbo sign. The cyst contents can be evacuated into the bronchial tree resulting in air-fluid level and crumpled endocyst floating in the cyst fluid (water lilly sign or camelotte sign). Although rare, we have seen calcification in one hydatid cyst and daughter cysts in another. Previous series have also documented the rare occurrence of cyst wall calcification or daughter cysts in pulmonary hydatid cysts. ${ }^{[11]}$ Ruptured hydatid cyst can have

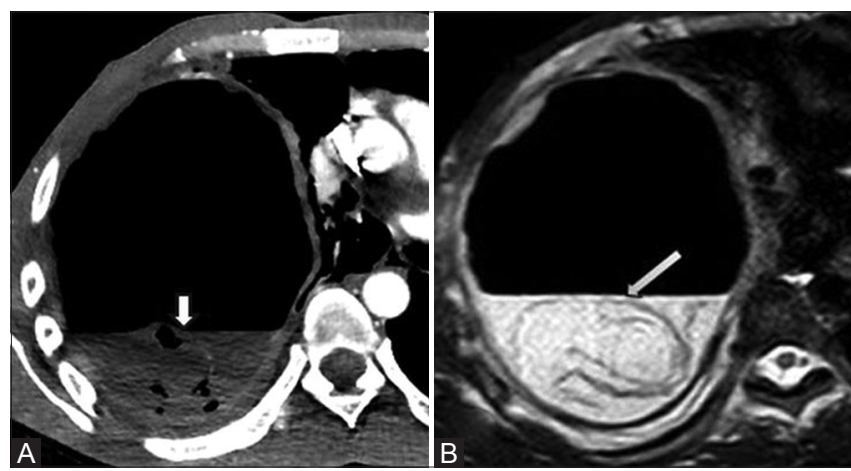

Figure 13 (A and B): Axial contrast-enhanced computed tomography image of the chest (A) showing a large cavity with air-fluid level, air bubble sign (white arrow), and suspicious folded membrane like structures. Axial T2-weighted magnetic resonance image (B) shows curvilinear hypointense membrane like structures (long white arrow) suggesting hydatid cyst. However, biopsy showed invasive aspergillosis

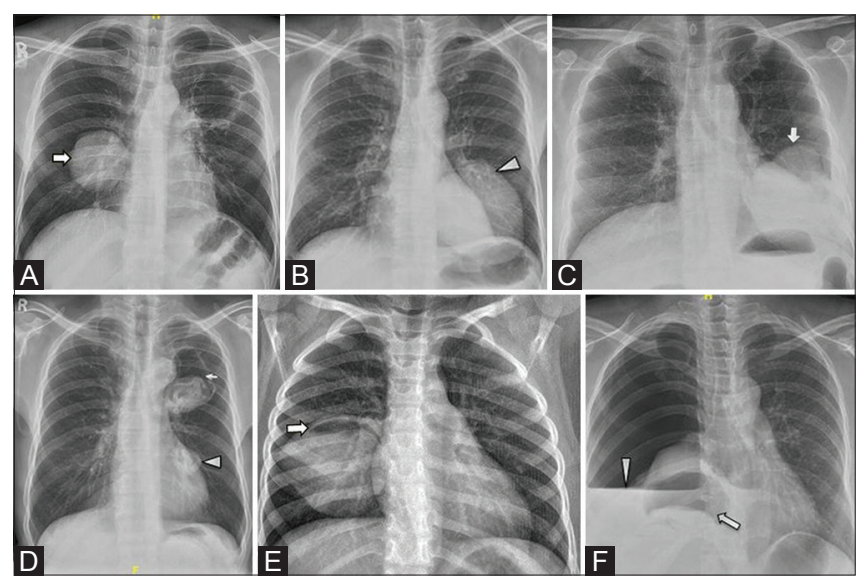

Figure $15(A-F)$ : Different chest radiograph findings of hydatid cysts, with round $(A)$, oval $(B)$, lobulated $(C)$ shapes, folded membranes (D), crescent sign $(E)$, and hydropneumothorax $(F)$. A ruptured hydatid cyst is also seen in the right lower lobe in $(F)$ with dependent folded membranes (white arrow in F). Another smaller cyst is seen in the left lung lower lobe in (D) with similar folded membranes (arrowhead in D)

air-fluid level, air bubbles or cavitation, and blurred margins mimicking a lung abscess, pneumonia, or inflammation on CT. ${ }^{[13]}$ Complicated cysts are also difficult to differentiate from other lesions, such as tumor, hematoma, or congenital cysts, pneumothorax. ${ }^{[13-16]}$

Varying MRI appearances of hydatid cyst have been documented. In the majority of previously reported cases, the cyst contents were hypointense on T1-weighted images and hyperintense on T2-weighted and proton density [PD] images ${ }^{[17-20]}$ with the capsule showing low signal intensity on T2-weighted images, which has been described as the hypointense rim sign, ${ }^{[18,20]}$ isointense signal on T1 weighted image, and mild post-contrast enhancement. However, there is no large study on the value of T2 hypointense rim in lung hydatid cysts. In our study, we found T2 hypointense rim in 2 unruptured cysts and T2 hypointense folded membranes in 18 ruptured cysts, as only atypical/doubtful cases underwent MRI. Hence, predictive values for each 
finding on MRI were not calculated. Further studies are needed to highlight the importance of $\mathrm{T} 2$ hypointense rim in lung hydatid cysts and to differentiate hydatid cysts from other lung cysts.

Other MRI sequences like diffusion weighted imaging (DWI), MR spectroscopy (MRS), and post-gadolinium images could also be used to evaluate hydatid cysts. Role of DWI has been studied in liver hydatid cysts, where significantly lower ADC values have been found in hydatid cysts in comparison with simple cysts. ${ }^{[21]}$ On comparing 27 hydatid cysts, 5 abscesses, and 5 simple cysts, Oruç et al. concluded that DWI has a potential role in classifying the types of hydatid cysts. In their study, type 1 hydatid cysts and simple cysts showed similar ADC values. Also, abscesses showed similar ADC values as type 4 hydatid cysts, but much lower ADC values than type 1 cysts. ${ }^{[22]}$ Pyruvate peak at $2.4 \mathrm{ppm}$ has been demonstrated on MRS of hydatid cysts in brain and has also been proposed to be a marker of viability. ${ }^{[23]}$ On post-contrast images, mild enhancement of the rim has been reported in hydatid cysts in different locations. ${ }^{[4]}$ In our study, we performed only T2W images and other MRI sequences were not acquired.

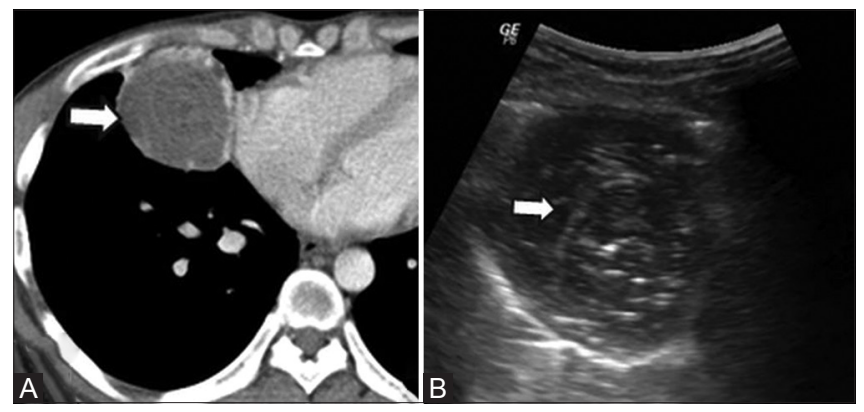

Figure 16 (A and $B$ ): Axial contrast-enhanced computed tomography of thorax (A) showing a well-defined peripherally located hypodense lesion with suspicious folded membranes in the right middle lobe (arrow). Ultrasound of the same patient (B) showing folded membranes in the hypoechoic lesion. Lesion proved to be a hydatid cyst after surgical excision
Further studies are warranted to evaluate the role of DWI, MRS, and post-gadolinium studies in differentiating lung hydatid cysts and their mimics.

In our study, the described typical CT signs helped in making a definite diagnosis of hydatid cyst in 52 patients having 60 cysts on CT. In 14 patients, the findings were atypical on $\mathrm{CT}$ and lung mass or abscess was considered as differential diagnoses. However, folded membranes were clearly demonstrated on MRI aiding accurate preoperative diagnosis of hydatid cyst in these patients. Similarly, solid-cystic appearance with absence of folded membranes on MRI in seven patients and cysts with absence of hypointense rim sign in three patients helped us to correctly offer an alternate diagnosis preoperatively. We encountered one false-positive case on MRI, where the debris in a fungal abscess resembled folded membranes. One hydatid cyst showed solid appearance and calcification on CT and did not show folded membranes or hypointense rim on MRI resulting in a false-negative diagnosis. We recommend the following imaging pathway for hypodense lesions seen on $\mathrm{CT}$, where hydatid cyst is suspected [Figure 17]. Certain limitations of our study include the retrospective nature and the fact that not all patients had MRI. Additional MRI was performed only when the findings were uncertain on CT.

\section{Conclusion}

Thick-walled unilocular cyst is the most common CT imaging appearance of pulmonary hydatid cysts. Ruptured or complicated hydatid cysts may show typical radiographic and CT appearances with many named imaging signs, pointing toward the diagnosis. However, some of the complicated cysts may be atypical on $\mathrm{CT}$, mimicking lung abscess or malignancy. In such situations, T2-weighted MRI can clearly demonstrate the ruptured folded membranes, clinching the diagnosis.

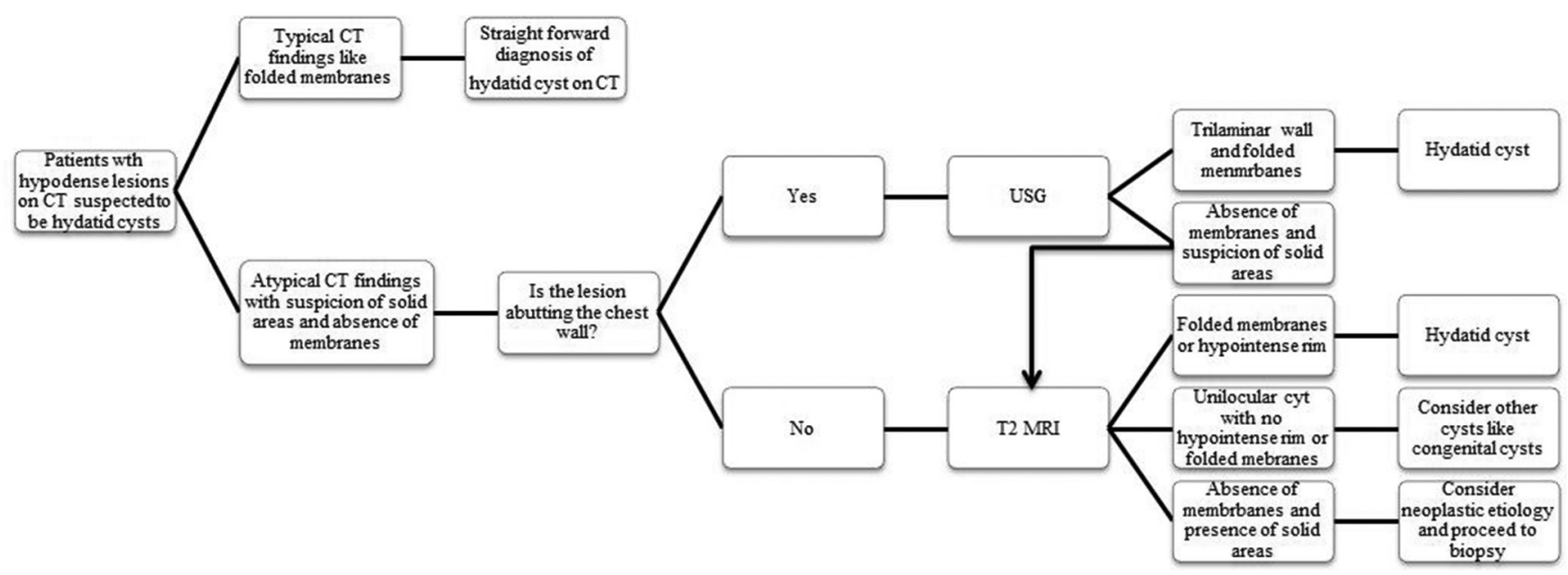

Figure 17: Proposed algorithm for imaging in suspected hydatid cyst 
Similarly, low-density tumors like schwannoma and malignant lesions like bronchogenic carcinoma and sarcoma can appear cystic on CT and mimic hydatid cysts. T2-weighted MRI can show that these lesions are actually solid with a "pseudocystic" appearance on CT, thus prompting biopsy. To conclude, we recommend additional T2-weighted MRI as a problem-solving tool to further characterize hypodense lesions with uncertain findings on CT.

\section{Financial support and sponsorship}

Nil.

\section{Conflicts of interest}

There are no conflicts of interest.

\section{References}

1. Singh N, Srinivas R, Bal A, Aggarwal AN. Lung carcinoma mimicking hydatid cyst: A case report and review of the literature. Med Oncol 2009;26:424-8.

2. Kilinç O, Döskaya M, Sakar A, Yorgancioğlu A, Halilçolar H, Caner A, et al. Three atypical pulmonary hydatidosis lesions mimicking bronchial cancer from Turkey. New Microbiol 2009;32:229-33.

3. Kabiri el H, Al Bouzidi A, Kabiri M. Askin tumor mimicking a hydatid cyst of the lung in children: Case report. Pan Afr Med J 2014;17:131.

4. Manson-Bahr PE, Apted FI. Manson's Tropical Diseases. London: Bailiere Tindall; 1982. p. 245-7.

5. Beggs I. The radiology of hydatid disease. AJR Am J Roentgenol 1985; 145:639-48.

6. Gottstein B, Reichen J. Hydatid lung disease (echinococcosis/ hydatidosis). Clin Chest Med 2002;23:397-408, ix.

7. Haliloglu M, Saatci I, Akhan O, Ozmen MN, Besim A. Spectrum of imaging findings in pediatric hydatid disease. AJR Am J Roentgenol 1997;169:1627-31.

8. Solak H, Yeniterzi M, Yüksek T, Anil N, Göktoğan T, Ceran S, et al. The hydatid cyst of the lung in children and results of surgical treatment. Thorac Cardiovasc Surg 1990;38:45-7.

9. Akgul Ozmen C, Onat S. Computed tomography (CT) findings of pulmonary hydatid cysts in children and the factors related to cyst rupture. Med Sci Monit 2017;23:3679-86.

10. Pedrosa I, Saíz A, Arrazola J, Ferreirós J, Pedrosa CS. Hydatid disease: Radiologic and pathologic features and complications. Radiographics 2000;20:795-817.

11. Jerray M, Benzarti M, Garrouche A, Klabi N, Hayouni A. Hydatid disease of the lungs. Study of 386 cases. Am Rev Respir Dis 1992;146:185-9.

12. Kervancioglu R, Bayram M, Elbeyli L. CT findings in pulmonary hydatid disease. Acta Radiol 1999;40:510-4.

13. Zhang Q, Huang TM, Li BZ, Li ZL, Liao KX. Misdiagnosis of pulmonary hydatid cyst rupture: Report of 38 cases. Zhonghua Jie He He Hu Xi Za Zhi 2003;26:474-6.

14. Von Sinner WN. Radiographic, CT and MRI spectrum of hydatid disease of the chest: A pictorial essay. Eur Radiol 1993;3:62-70.

15. Kayhan S, Sahin U, Turut H, Yurdakul C. An unusual radiological presentation of a pulmonary hydatid cyst in a child. J Clin Imaging Sci 2013;3:20.

16. Poyrazoglu H, Tor F, Avsar MK, Bayraktar I, Ulus T. An unusual approach to a ruptured hydatid cyst case which had been treated with a wrong diagnosis. Indian J Thorac Cardiovasc Surg 2008;24:22-3.

17. Singh S, Gibikote SV. Magnetic resonance imaging signal characteristics in hydatid cysts. Australas Radiol 2001;45:128-33.

18. Kalovidouris A, Gouliamos A, Vlachos L, Papadopoulos A, Voros D, Pentea S, et al. MRI of abdominal hydatid disease. Abdom Imaging 1994;19:489-94.

19. Marani SA, Canossi GC, Nicoli FA, Alberti GP, Monni SG, Casolo PM, et al. Hydatid disease: MR imaging study. Radiology 1990;175:701-6.

20. von Sinner WN, Rifai A, te Strake L, Sieck J. Magnetic resonance imaging of thoracic hydatid disease. Correlation with clinical findings, radiography, ultrasonography, CT and pathology. Acta Radiol 1990;31:59-62.

21. Inan N, Arslan A, Akansel G, Anik Y, Sarisoy HT, Ciftci E, et al. Diffusion-weighted imaging in the differential diagnosis of simple and hydatid cysts of the liver. AJR Am J Roentgenol 2007;189:1031-6.

22. Oruç E, Yıldırım N, Topal NB, Kılıçturgay S, Akgöz S, Savcı G, et al. The role of diffusion-weighted MRI in the classification of liver hydatid cysts and differentiation of simple cysts and abscesses from hydatid cysts. Diagn Interv Radiol 2010;16:279-87.

23. Jayakumar PN, Srikanth SG, Chandrashekar HS, Kovoor JM, Shankar SK, Anandh B, et al. Pyruvate: An in vivo marker of cestodal infestation of the human brain on proton MR spectroscopy. J Magn Reson Imaging 2003;18:675-80.

24. García-Díez AI, Ros Mendoza LH, Villacampa VM, Cózar M, Fuertes MI. MRI evaluation of soft tissue hydatid disease. Eur Radiol 2000;10:462-6. 